\title{
Assessing readiness for change in the Helmet use: a motivational interview
}

\author{
Fardin Shahbazzadeh ${ }^{1}$, Sajjad Narimani2 ${ }^{*}, S_{i v a}$ Ahmadi ${ }^{1}, Z a h r a ~ A n v a r i{ }^{1}$, Mehdi Khezeli $^{3}$ \\ 'Students Research Committee, School of Meshkin Nursing, Ardabil University of Medical Sciences, Ardabil, Iran \\ ${ }^{2}$ Social Determinant of Health Research Center, Ardabil University of Medical Sciences, Ardabil, Iran \\ ${ }^{3}$ Social Development and Health Promotion Research Center, Health Institute, Kermanshah University of Medical Sciences, \\ Kermanshah, Iran
}

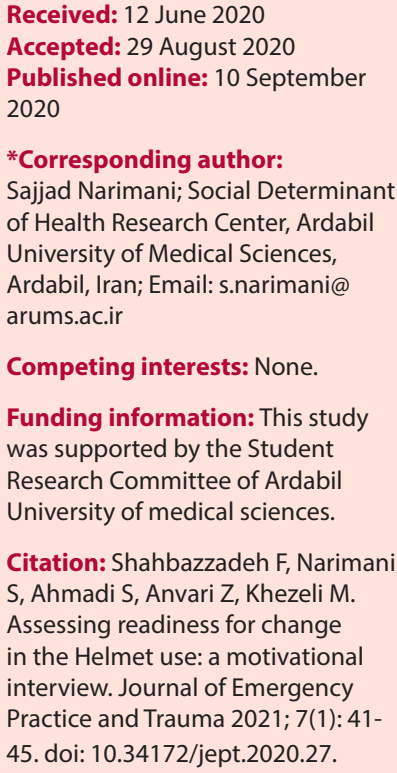

\begin{abstract}
Objective: This study aimed to determine the motivation to helmet use among motorcyclists in Ardabil city using the trans-theoretical model (TTM) motivational interview.

Methods: Random cluster sampling was used for this study. Five gas stations were selected from the crowded areas of Ardabil city and motorcyclists who came to these places were randomly interviewed. In this descriptive cross-sectional study, 350 motorcyclists in Ardabil were selected and interviewed by TTM Motivational questionnaire. We used one-way analysis of variance (ANOVA) and linear regression for analysis of data in SPSS software version 20. Results: Findings revealed that $12.6 \%$ of respondents consistently wore a helmet when they rode a motorcycle, while $45.2 \%$ of helmet non-wearers were uncertain about wearing a helmet in the future. Decisional balance (pros), self-efficacy (decreasing the negative affect situation score), and behavioral process of change can motivate participants to helmet use. Conclusion: Reducing negative social situations and increasing positive emotional states can motivate people to use helmet.
\end{abstract}

Keywords: Helmet, Motivational interview, Readiness for change

\section{Introduction}

Iran faces road safety challenges. The country has a high rate of road traffic deaths, with 32.30 deaths per 100000 population per year according to the World Health Organization (WHO) estimate in 2018. About 6000 traffic accidents occur annually in the country; killing 43 people daily. Motorcyclists mortality accounted for $21.8 \%$ of total traffic accidents $(1,2)$. Most motorcycle fatalities occur on suburban roads that account for $44.5 \%$ of the total mortality. Subsequently, $38.5 \%$ died in urban areas, $10.8 \%$ in rural areas, $1.2 \%$ in private roads, and $5.2 \%$ in other areas. Statistics show that the most common cause of death for motorcyclists is injuries to the head that was responsible for $51.6 \%$ of deaths. Based on evidence, $54.6 \%$ of motorcycle fatalities were among people aged between 18 and 39 years. A significant percentage of motorcycle crashes happen among teenagers and adolescents (24). This can be due to many factors such as type of the vehicle, easier procurement, the excitement of using it, age condition, non-compliance with laws and regulations, risky driving, non-use of helmet, and having more passengers than allowed capacity. In this regard, the best action to prevent the mortality is the use of helmet. Although a large proportion of accidents are directly related to the attitude and behavior of individuals, the literature shows that despite the importance of this issue, there are ample studies using theories and models of behavior change to investigate risk taking behaviors. (5-11). It seems that the application of behavioral science to injury prevention has lingered behind other approaches in the history of injury prevention efforts. Without a theory-driven approach, many of these interventions failed or attained limited success $(8,9)$. The failure to use health behavior theories to understand behavioral health factors and to develop effective interventions may be a core factor behind the limited success of injury prevention efforts to change behaviors. Health behavior theories offer important insights into behavioral change, and are most applicable at different levels of risk taking behaviors. Specifically, the intrapersonal level of influence focuses on the impact 
of an individual's knowledge, beliefs, and attitudes on behavior. Health behavior theories that emphasize cognition, motivation, and perception are most applicable at this level (9). One of the health education models that can be used to assess motivation of participants to apply healthy behavior is the trans-theoretical model (TTM). This model of behavior change is an integrative theory of therapy that assesses an individual's readiness to act on a new healthier behavior, and provides strategies, or processes of change to guide the individual. The model is composed of constructs such as: stages of change, processes of change, self-efficacy, and decisional balance (9). The purpose of this study was to determine the motivation of helmet use among motorcyclists in Ardabil city using the TTM Motivational Questionnaire.

\section{Methods}

In this descriptive cross-sectional study, motivational interviews for helmet use based on TTM questionnaire were performed in Ardabil city. To obtain valid results, we first prepared the list of registered motorcyclists through the police station according to the statistical population which consisted of 3750 motorcyclists. Due to the fact that the prior studies have shown different results concerning the use of helmet among motorcyclists $(3,4)$, we decided to use the default value for $\mathrm{p}$ and $\mathrm{q}$ in Cochran's formula ( $\mathrm{p}$ $=0.5, q=0.5, \alpha=0.95, d=0.05$ ) and the sample size was estimated to be 350 for the current study.

$$
n=\frac{\mathrm{Z}^{2} p(1-p)}{\mathrm{d}^{2}}
$$

Random cluster sampling was used for this study. Among 61 gas stations in five regions including north, south, east, west and the center of Ardabil city, five gas stations were selected from the crowded areas of these clusters. Then, the motorcyclists who came to these places were randomly interviewed. After obtaining written informed consent from participants, we initiated the motivational interviews using TTM questionnaire. The questionnaire included questions about motorcycle riding behaviors and experiences (frequency and distance of motor rides, reason and location for motor rides, and history of motor rides) and four different measures regarding helmet-use behaviors: A Stage of Change (SOC) measure (see Supplementary file 1), a Decisional Balance measure (pros and cons of wearing a motorcycle helmet) (see Supplementary file 1), a self- efficacy measure (positive affect situation, negative affect situation and habit situation) (see Supplementary file 1), and a Process of Change (POC) measure including cognitive and behavioral processes (see Supplementary file $1(8,9)$. In this study, the scales were translated into Persian and then were back-translated into English. The content validity of the questionnaire was measured using content validity ratio (CVR) and content validity index (CVI) via an expert panel including eight health education specialists and two epidemiologists. Validity of the questionnaires was confirmed by the CVI of $96 \%$, $90 \%, 82 \%$, and $87 \%$, and CVR of $96 \%, 88 \%, 90.6 \%$, and $88 \%$ for SOC, decisional balance, self-efficacy, and POC scales, respectively. The final version of the questionnaire was pilot tested with 40 motorcyclists using test-retest measurement. Spearman-Brown correlation coefficients to determine the test-retest reliability were $87.5 \%, 80 \%$, $75 \%$ and $74 \%$ for SOC, decisional balance, self-efficacy, and POC scales, respectively.

\section{Results}

Participant $(\mathrm{N}=350)$ were placed in five stages of behavior change based on self-reported helmet use behavior, in answer to the question: Do you always wear a helmet when you ride a motorcycle? Findings showed that $12.6 \%$ of respondents in maintenance and action stages consistently wore a helmet when they rode a motorcycle, while $42.3 \%$ of people who were in the precontemplation stage did not think about wearing a helmet at all and had no intrinsic motivation to wear a helmet in the future (see Figure 1).

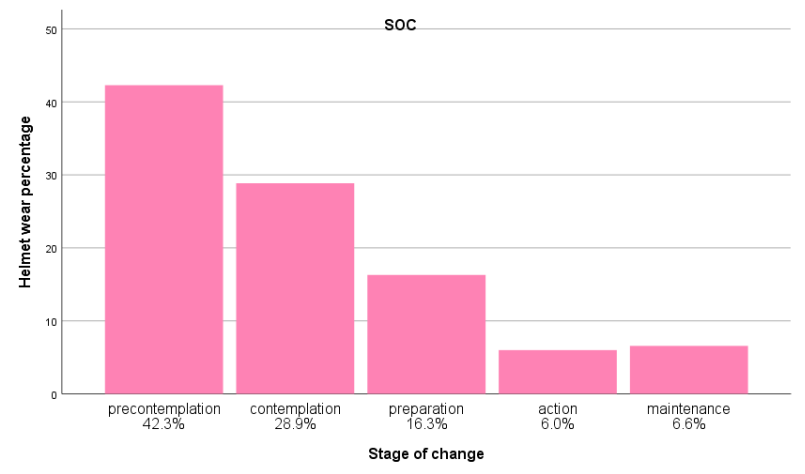

Figure 1. Distribution of participants across stages of behavior change.

Table 1. One-way ANOVA to compare "pros" scores across stages of change

\begin{tabular}{|c|c|c|c|c|c|c|}
\hline \multirow{2}{*}{ Symbol } & \multirow{2}{*}{ Decision balance questions (pros) } & \multirow{2}{*}{ Mean } & \multirow{2}{*}{ SD } & \multirow{2}{*}{$P$ value } & \multicolumn{2}{|c|}{$95 \% \mathrm{Cl}$ for Mean } \\
\hline & & & & & Lower bound & Upper bound \\
\hline DB1 & Wearing a helmet is a good choice & 3.95 & 1.16 & $0.016^{*}$ & 0.07 & 3.80 \\
\hline DB2 & Smart riders wear helmets & 4 & 1.12 & $0.015^{*}$ & 0.06 & 3.86 \\
\hline DB3 & Helmet decreases head injuries & 4.33 & 1.07 & $0.009^{*}$ & 0.06 & 4.21 \\
\hline DB4 & Helmet protects me on roads shared with cars & 4.10 & 1.10 & $0.021^{*}$ & 0.06 & 3.97 \\
\hline DB5 & I feel safer when I wear a helmet while riding a motorcycle & 3.90 & 1.18 & $0.004^{*}$ & 0.07 & 3.76 \\
\hline
\end{tabular}

* Significant at the 0.05 level 


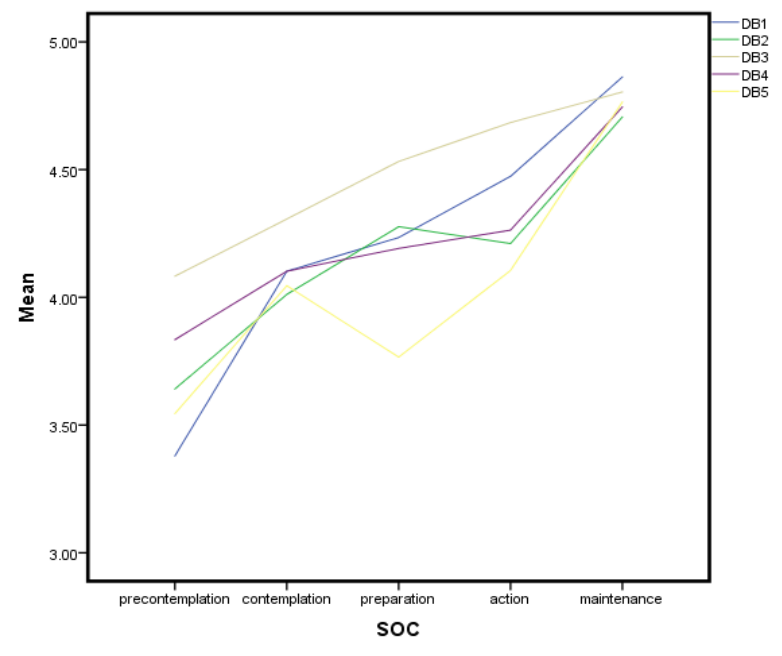

Figure 2. comparing score of DB1 (wearing a helmet is a good choice), DB2 (smart riders wear helmets), DB3 (helmet decreases head injuries), DB4 (Helmet protects me on roads shared with cars), and DB5 (I feel safer when I wear a helmet while riding a motorcycle) across different stages of change.

Table 1 and Figure 2 show the results of one-way analysis of variance (ANOVA) between the stages of change and decisional balance construct (pros) at 5\% level of significance. The results indicated that DB2 and DB3 were positively and significantly correlated with the stages of change, and were the strongest motivator to transition from precontemplation and contemplation stages to action and maintenance stages. These results imply that the motorcyclists strongly believe that "smart riders wear helmets" and "helmet decreases head injuries". Hence, pros were predictors and motivators of helmet use.

Table 2 and Figure 3 show the correlation between the stages of change and the temptation scale (negative affect situation) at $5 \%$ level of significance. The results indicated that $7^{\text {th }}$ and $8^{\text {th }}$ questions of self-efficacy including: (when I am stressed) and (when I am nervous) were significantly correlated with the stages of change, and were motivators to transition from precontemplation and contemplation to preparation stage. Also, $6^{\text {th }}$ question of self-efficacy (when I am worried about something) was the strongest motivator to stage transition across the stage of change. Results also showed that when temptation score decreased, self-efficacy score increased.

As shown in Table 3, the results of one-way ANOVA to compare the mean score of processes of change showed that the mean score of cognitive processes (with the exception

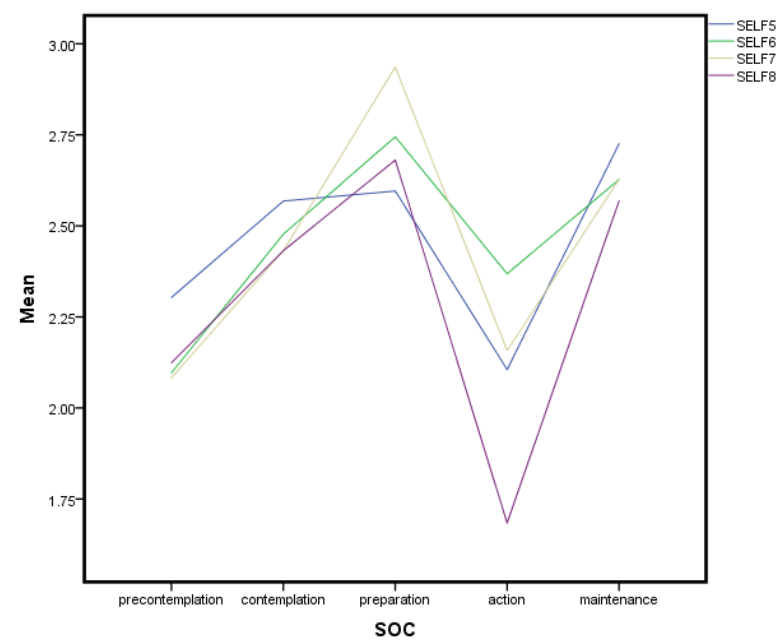

Figure 3. Comparing the score of negative affect situations $\left(5^{\text {th }}\right.$ to $8^{\text {th }}$ questions of self-efficacy scale) across stages of change.

of environmental re-evaluation) was significantly different in the five stages of behavior change. Also, the mean scores of all behavioral processes were significantly different during the stages of behavior change.

Ordinal regression showed that "pros" as a sub-construct of decisional balance was the strongest motivator of stage transition to helmet use, then negative affect situation and self-liberation (behavioral process of change) were next significant motivators, respectively. This result indicated that increasing pros, and self-liberation, and decreasing the negative affect situation can improve the motivation to helmet use in motorcyclists of Ardabil city (see Table 4).

\section{Discussion}

The WHO considers helmet use as a protective tool against head injury amongst motorcyclists $(1,12-14)$. Despite the explicit benefits of the helmet in reducing casualties and mortality from head injuries, helmet non-usage is still on a rise and is responsible for head injuries and fatalities amongst motorcycle riders (15). The present study aimed to investigate readiness for change, and motivators of helmet use among motorcyclists in Ardabil city. The results of this study support the application of motivational interview and transtheoretical model of behavior change to conceptualize and assess the helmet use as well as the design and implementation of interventions to promote

Table 2. One-way ANOVA to compare self-efficacy scores (negative affect situations) across stages of change

\begin{tabular}{|c|c|c|c|c|c|}
\hline \multirow{2}{*}{ Negative effect situations } & \multirow{2}{*}{ Mean } & \multirow{2}{*}{ SD } & \multirow{2}{*}{$P$ value } & \multicolumn{2}{|c|}{$95 \% \mathrm{Cl}$ for Mean } \\
\hline & & & & Lower bound & Upper Bound \\
\hline When I feel angry or depressed. & 2.46 & 1.13 & 0.28 & 0.07 & 2.31 \\
\hline When I am worried about something. & 2.37 & 1.22 & $0.007^{* *}$ & 0.06 & 2.23 \\
\hline When I am stressed. & 4.33 & 1.37 & $0.001^{* *}$ & 0.07 & 2.22 \\
\hline When I am nervous. & 2.36 & 1.24 & $0.05^{*}$ & 0.07 & 2.16 \\
\hline
\end{tabular}

**Significant at the 0.01 level

*Significant at the 0.05 level 
Table 3. One-way ANOVA to compare "processes of change" scores across stages of change

\begin{tabular}{|c|c|c|c|c|c|c|}
\hline & \multirow{2}{*}{ Process of change } & \multirow{2}{*}{ Mean } & \multirow{2}{*}{ SD } & \multirow{2}{*}{$P$ value } & \multicolumn{2}{|c|}{$95 \% \mathrm{Cl}$ for Mean } \\
\hline & & & & & Lower bound & Upper bound \\
\hline \multirow{5}{*}{ Cognitive } & Consciousness Raising & 10.26 & 3.23 & $0.009 * *$ & 0.17 & 9.92 \\
\hline & Dramatic Relief & 9.53 & 3.61 & $0.070^{*}$ & 0.19 & 9.15 \\
\hline & Environmental Reevaluation & 10.16 & 3.32 & 0.294 & 0.17 & 9.81 \\
\hline & Self-Reevaluation & 9.88 & 3.35 & $0.008^{* *}$ & 0.17 & 9.53 \\
\hline & Social Liberation & 9.50 & 2.85 & $0.042^{*}$ & 0.15 & 9.20 \\
\hline \multirow{5}{*}{ Behavioral } & Counter conditioning & 9.36 & 3.22 & $0.011^{*}$ & 0.17 & 9.02 \\
\hline & Helping Relationships & 7.30 & 3.03 & $0.032^{*}$ & 0.16 & 6.98 \\
\hline & Self-Liberation & 9.75 & 3.29 & $0.023^{*}$ & 0.17 & 9.40 \\
\hline & Stimulus Control & 9.29 & 3.15 & $0.009^{* *}$ & 0.16 & 8.95 \\
\hline & Reinforcement Management & 9.80 & 3.22 & $0.001^{* *}$ & 0.17 & 9.46 \\
\hline
\end{tabular}

**Significant at the 0.01 level

*Significant at the 0.05 level

Table 4. Predictors of stage transition to helmet use in motorcyclists of Ardabil city

\begin{tabular}{lcccc}
\hline Ordinal regression & $\boldsymbol{\beta}$ & SE & Wald & $\boldsymbol{P}$ value \\
\hline Pros & 0.19 & 0.024 & 19.21 & 0.001 \\
negative affect situation & 0.18 & 0.022 & 9.95 & 0.002 \\
self-liberation & 0.17 & 0.036 & 4.75 & 0.029 \\
\hline
\end{tabular}

helmet use in motorcyclists. This study incorporated a multidimensional model to apply all constructs of the TTM (SOC, decisional balance, self-efficacy, and processes of change) to better understand the motivation of motorcycle helmet use and behavior change (9). This study provides important information about factors that may have an impact on helmet use behaviors. These factors include the pros (a sub-construct of decisional balance), feelings of confidence to wear a helmet and temptation not to wear a helmet (negative affect situations), use of cognitive and behavioral processes to successfully implement helmetrelated behavior change. These results are similar to the findings when the TTM is applied to other health-related behaviors $(8,10,11,16)$. Pros were the strongest motivator in the sample. The majority of participants in response to the following questions: "smart riders wear helmets" and "helmets decrease head injuries" answered (very important $=5$ ). Therefore, considering the perceived benefits of helmet use amongst participants, conducting an intervention based on the theories and models of health education and promotion can have positive results in order to increase the use of helmets in motorcyclists in Ardabil city. These results are consistent with the findings of other studies (10-13). When "negative affect situation" was controlled, the motivation to behavior change across stage of change was increased, which is consistent with the study of Hammond and Hall (8). Behavioral processes of change (such as self-liberation and stimulus control) were other motivators and predictors of helmet use, therefore training and disseminating rules and stimulators which can remind helmet use to motorcyclists is recommended. This finding is in line with other health-related studies $(17,18)$. Similar with the results of other health-related studies (17-20), counter conditioning as a behavioral process of change (e.g. instead of wearing a hat or nothing

on my head when I ride a motorcycle) was a motivator to start helmet use, therefore the theory-based education can focus on this sub-construct to motivate motorcyclists to use helmets. Similar to other health-related studies (8,17-20), other behavioral processes including "helping relationship", and, "reinforcement management" are other strong motivators which keep motorcyclists in action stage and conduct to maintenance stage.

\section{Conclusion}

It can be concluded that increasing the pros, decreasing temptation (negative affect), and promoting behavioral processes of change can be used to motivate motorcyclists to think about helmet and finally wearing while riding a motorcycle. This study indicates that the application of motivational interviewing techniques can effectively support stage progression in helmet use and integrating MI techniques into helmet-use interventions can lead to the behavior change in order to increase wearing the helmet among motorcyclists.

\section{Limitations}

The results suggest caution in over-reliance on self-report data. Also, participants in this research may have overreported their actual rate of helmet use. However, street observations along with participant self-reporting were conducted to further validate the results.

\section{Authors' Contributions}

FS : Sampling and motivational interview

$\mathrm{SA}$ and ZA : help to motivational interview

SN: study design and Spss Analysing And manuscript Writing

MK: questionnaire Reliability and Validity and help to Writing manuscript

\section{Ethical issues}

Approval for all procedures was obtained from the ethics committee of Ardabil University of medical sciences (ethics No. IR.ARUMS.REC.1398.194). In addition, we received permission from Ardabil police to conduct this study. 


\section{Supplementary files}

Supplementary file 1 contains four different measures regarding helmet-use behaviors.

\section{References}

1. World Health Organization (WHO). Global Status Report on Road Safety. WHO; 2018.

2. Barzegar A, Ghadipasha M, Forouzesh M, Valiyari S, Khademi A. Epidemiologic study of traffic crash mortality among motorcycle users in Iran (2011-2017). Chin J Traumatol. 2020;23(4):219-223. doi:10.1016/j. cjtee.2020.05.008.

3. Yadollahi M, Gholamzadeh S. Five-year forecasting deaths caused by traffic accidents in Fars province of Iran. Bull Emerg Trauma 2019; 7(4): 373-80. doi: 10.29252/beat070406.

4. Azami-Aghdash S, Sadeghi-Bazargani H, Shabaninejad $\mathrm{H}$, Abolghasem Gorji H. Injury epidemiology in Iran: a systematic review. J Inj Violence Res 2017; 9(1): 27-40. doi: 10.5249/jivr.v9i1.852.

5. Trifiletti LB, Gielen AC, Sleet DA, Hopkins K. Behavioral and social sciences theories and models: are they used in unintentional injury prevention research? Health Educ Res 2005; 20(3): 298-307. doi: 10.1093/her/cyg 126.

6. Bakhtiyari M, Mehmandar MR, Khezeli M, Latifi A, Jouybari TA, Mansournia MA. Estimating the avoidable burden and population attributable fraction of human risk factors of road traffic injuries in iran: application of penalization, bias reduction and sparse data analysis. Int J Inj Contr Saf Promot 2019; 26(4): 405-11. doi: 10.1080/17457300.2019.1660374.

7. Rezapur-Shahkolai F, Khezeli M, Hazavehei SM, Ariapooran S, Soltanian AR, Ahmadi A. The effects of suicidal ideation and constructs of theory of planned behavior on suicidal intention in women: a structural equation modeling approach. BMC Psychiatry 2020; 20(1): 217. doi: 10.1186/ s12888-020-02625-w.

8. Hammond J, Hall S. Using the Transtheoretical Model of Behavior Change to Increase Bicycle Helmet Use. Presented at the annual convention of the International Neuropsychological Society; 2015; Seattle, WA:

9. Glanz K, Rimer BK, Viswanath K. Health Behavior and Health Education: Theory, Research, and Practice. 4th ed. Jossey-Bass; 2008.

10. Narimani S, Farmanbar R, Kazemnejad Leyli E. Predictors of intention to quit smoking among hospital male staff.
Journal of Research and Health 2017; 7(2): 674-81.

11. Narimani S, Farmanbar R, Kazemnejad E, Hooshmandi A. Mediating Effects of Self-Efficacy in the Transtheoretical Model among Hospital Male staff of Ardebil University of Medical Sciences in 2014. Biological Forum 2015; 7(2): 806-811.

12. World Health Organization (WHO). Helmets: A Road Safety Manual for Decision-Makers and Practitioners. Geneva, Switzerland: WHO; 2006.

13. Keng SH. Helmet use and motorcycle fatalities in Taiwan. Accid Anal Prev 2005; 37(2): 349-55. doi: 10.1016/j. aap.2004.09.006.

14. Li LP, Li GL, Cai QE, Zhang AL, Lo SK. Improper motorcycle helmet use in provincial areas of a developing country. Accid Anal Prev 2008; 40(6): 1937-42. doi: 10.1016/j.aap.2008.06.019.

15. Adewoye KR, Aremu SK, Olomofe CO, Adeniyi AM, Agbana $\mathrm{RD}$, Abioye OO, et al. The prevalence and determinants of helmet use amongst commercial motorcyclists in Ido-Osi local government area. Arch Environ Occup Health 2020; 75(6): 358-64. doi: 10.1080/19338244.2019.1673692.

16. Armitage CJ, Sheeran P, Conner M, Arden MA. Stages of change or changes of stage? Predicting transitions in transtheoretical model stages in relation to healthy food choice. J Consult Clin Psychol 2004; 72(3): 491-9. doi: 10.1037/0022-006x.72.3.491

17. Narimani S, Farmanbar R, Mozaffari N, Nemati A, Amani F, Abbasgholizadeh N. Cognitive and behavioral processes and its stages of change for fruit and vegetable consumption among nursing and midwifery staff of Ardabil hospitals, 2016: application of the trans theoretical model. Journal of Health 2018; 9(2): 182-95. doi: 10.29252/j.health.9.2.182. [In Persian].

18. Khosravi A, Emamian MH, Hashemi H, Fotouhi A. Transition in tobacco use stages and its related factors in a longitudinal study. Environ Health Prev Med 2018; 23(1): 39. doi: 10.1186/s12199-018-0728-x.

19. Suh YS, Chung YJ. The effect of nutrition education on the improvement of psychosocial factors related to vegetable and fruit intake of elementary school children in pre-action stages. Korean J Nutr 2009: 43(6):597-606. doi: 10.4163/ kjn.2010.43.6.597.

20. Davidson TL, Jones S, Roy M, Stevenson RJ. The cognitive control of eating and body weight: it's more than what you "think". Front Psychol 2019; 10: 62. doi: 10.3389/ fpsyg.2019.00062. 